\title{
Pseudomelanosis ilei associated with ingestion of oral iron therapy
}

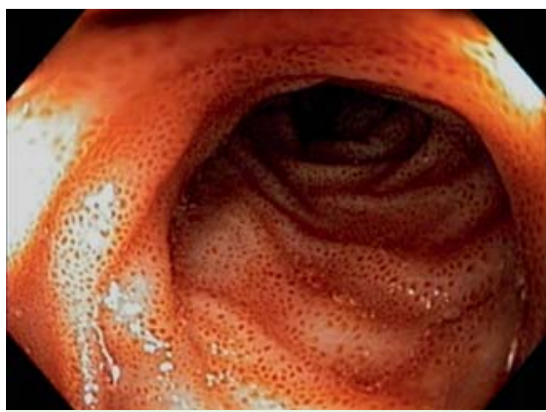

Fig. 1 Colonoscopy with terminal ileum intubation showing the speckled brown hyperpigmentation of the terminal ileum.

A 63-year-old man was seen in consultation for iron deficiency anemia without overt bleeding. Other problems included depression, hypertension, and aortic valve insufficiency. Medications included escitalopram oxalate, metoprolol, and furosemide. Upper endoscopy with small-bowel biopsies was normal. Colonoscopy revealed a $1.5-\mathrm{cm}$ sessile polyp in the descending colon, which was removed with hot snare polypectomy. Examination of the terminal ileum was unremarkable. Histopathology revealed villous adenoma with low grade dysplasia. The patient began therapy with ferrous sulfate for his iron deficiency. His anemia resolved and his iron counts returned to normal.

Surveillance colonoscopy 1 year later revealed no more polyps. The terminal ileum revealed a speckled pattern of brown hyperpigmentation beginning from the ileocecal valve and extending up to $10 \mathrm{~cm}$ proximally ( Fig. 1).

Biopsy revealed brown pigment deposition within macrophages in the lamina propria of normal villi ( $\bullet$ Fig. 2 ).

A positive Prussian blue stain indicated hemosiderin deposition ( $\bullet$ Fig. 3 ).

HMB-45 staining for melanoma was unremarkable. Capsule endoscopy was done to assess the remaining small bowel. It appeared normal except for findings in the terminal ileum, which were identical to the colonoscopy findings ( $\bullet$ Fig. 4).

As the anemia had resolved, iron therapy was discontinued. Follow-up hemoglobin and iron studies 6 months later remained normal.
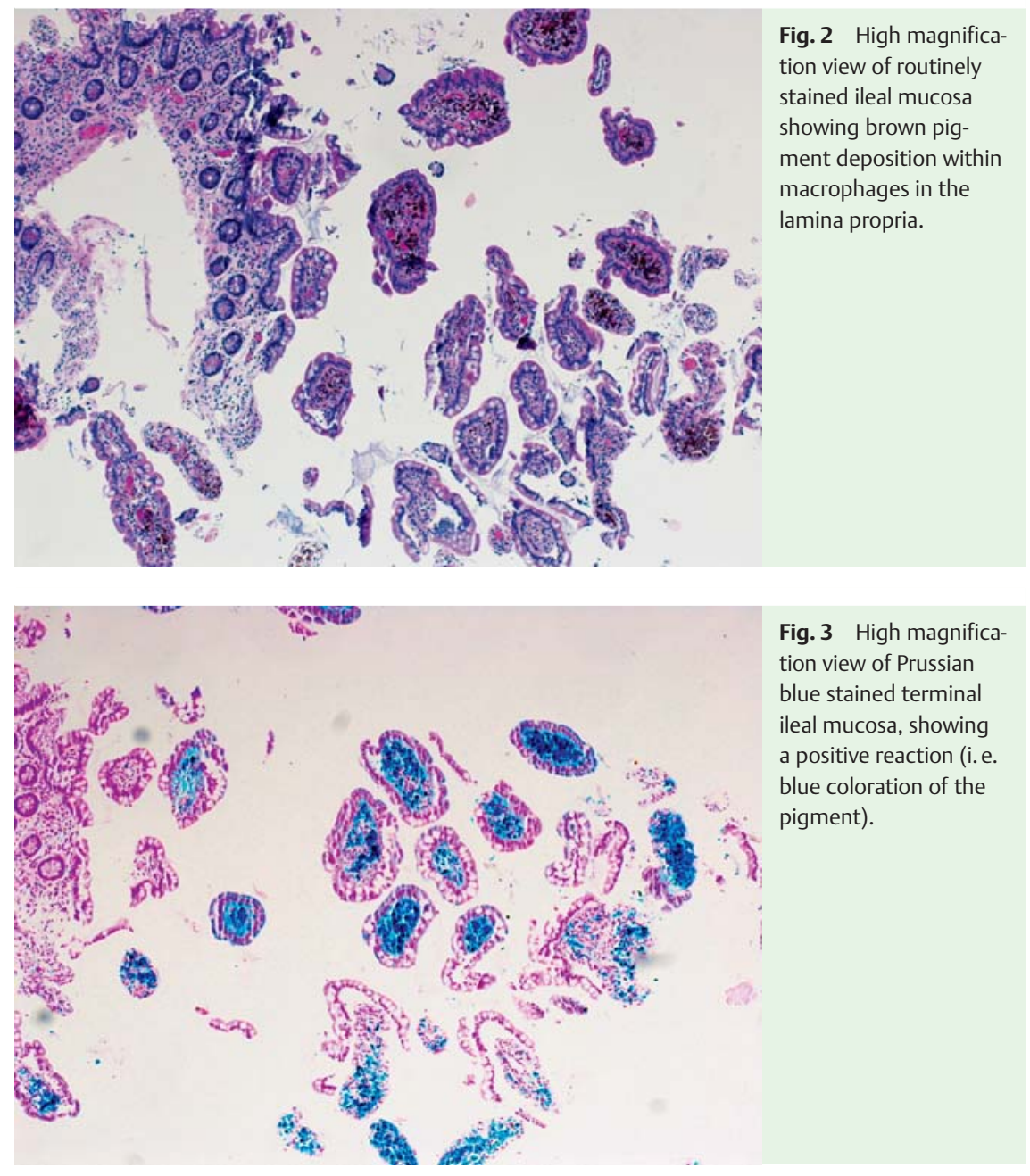

Pseudomelanosis of the gastrointestinal tract has been described from the esophagus through to the colon. Pseudomelanosis isolated to the ileum is extremely rare and the literature is limited to a few case reports [1,2]. Iron deposition has been associated with gastrointestinal bleeding, hemochromatosis, chronic renal failure, enteric iron, and several antihypertensive medications [3]. This case report strengthens the association between pseudomelanosis of the small bowel and oral iron therapy. Although a rare entity, physicians should be aware of it to facilitate a prompt diagnosis and avoid unnecessary testing.

\section{Competing interests: None}

Endoscopy_UCTN_Code_CCL_1AD_2AJ

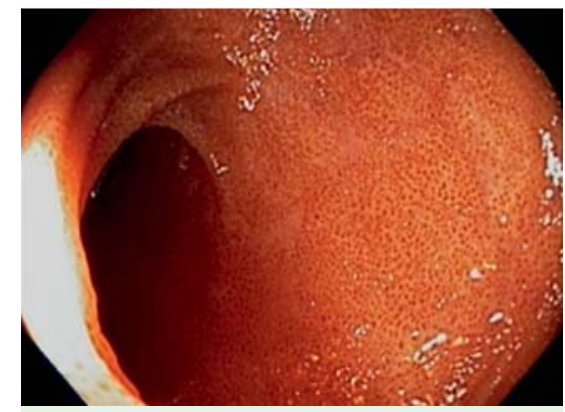

Fig. 4 Capsule endoscopy showing the speckled brown hyperpigmentation of the terminal ileum.

\section{R. Kibria, S. A. Ali, S. Akram}

Gastroenterology, Wright State University School of Medicine, Dayton, Ohio, USA 


\section{References}

1 Moore JD, Baichi M, Toledo R, Sitrin M. Pseudomelanosis of jejunum and ileum. Gastrointest Endosc 2007; 66: 857 - 859

2 Ghadially FN, Boone SA, Walley VM. Melanosis (haemosiderosis) ilei. J Submicrosc Cytol Pathol 1994; 26: 461 - 465

3 Almeida N, Figueiredo P, Lopes S. Small bowel pseudomelanosis and oral iron therapy. Dig Endosc 2009; 21: 128-130

\section{Bibliography}

Dol 10.1055/s-0030-1255603

Endoscopy 2010; 42: E243-E244

(c) Georg Thieme Verlag KG Stuttgart · New York . ISSN 0013-726X
Corresponding author

R. Kibria, MD

Gastroenterology

Wright State University School of Medicine 4100 West Third Street

Dayton

Ohio 45428

USA

Fax: +1-937-268-4737

rekibria@gmail.com 